CERN-TH/95-222

LPTENS-95/40

hep-th/9509017

\title{
One-Loop Calculation of Coupling Constants in IR regulated String Theory
}

\author{
Elias Kiritsis and Costas Kounnasin \\ Theory Division, CERN, \\ CH-1211, Geneva 23, SWITZERLAND
}

\begin{abstract}
Exact Superstring solutions are constructed moving in 4-D space-time with positive curvature and non-trivial dilaton and antisymmetric tensor fields. The full spectrum of string excitations is derived as a function of moduli fields $T^{i}$ and the scale $\mu^{2}=1 /(k+2)$ which induced by the non-zero background fields. The spectrum of string excitations has a non-zero mass gap $\mu^{2}$ and in the weak curvature limit ( $\mu$ small) $\mu^{2}$ plays the role of a well defined infrared regulator, consistent with modular invariance, gauge invariance, supersymmetry and chirality.

The effects of a covariantly constant chomo-magnetic field $B$ as well as additional curvature can be derived exactly up to one string-loop level. Thus, the one-loop corrections to all couplings (gravitational, gauge and Yukawas) are unambiguously computed and are finite both in the Ultra-Violet and the Infra-Red regime. These corrections are necessary for quantitative string superunification predictions at low energies. The one-loop corrections to the couplings are also found to satisfy Infrared Flow Equations.
\end{abstract}

CERN-TH/95-222

August 1995

*To appear in the proceedings of the Trieste Spring School and Workshop on String Theory, 27 March-7 April 1995.

†On leave from Ecole Normale Supérieure, 24 rue Lhomond, F-75231, Paris, Cedex 05, FRANCE. 


\section{Introduction}

Four-dimensional superstring solutions in a flat background [1]-[7] define, at low energy, effective supergravity theories [8]-[11]. A class of them successfully extends the validity of the standard model up to the string scale, $M_{s t r}$. The first main property of superstrings is that they are ultraviolet-finite theories (at least perturbatively). Their second important property is that they unify gravity with all other interactions. This unification does not include only the gauge interactions, but also the Yukawa ones as well as the interactions among the scalars. This String Unification happens at large energy scales $E_{t} \sim \mathcal{O}\left(M_{s t r}\right) \sim$ $10^{17} \mathrm{GeV}$. At this energy scale, however, the first excited string states become important and thus the whole effective low energy field theory picture breaks down [12]-[15]. Indeed, the effective field theory of strings is valid only for $E_{t} \ll M_{s t r}$ by means of the $\mathcal{O}\left(E_{t} / M_{s t r}\right)^{2}$ expansion. It is then necessary to evolve the String Unification predictions to a lower scale $M_{U}<M_{s t r}$ where the effective field theory picture makes sense. Then, at $M_{U}$, any string solution provides non-trivial relations between the gauge and Yukawa couplings, which can be written as

$$
\frac{k_{i}}{\alpha_{i}\left(M_{U}\right)}=\frac{1}{\alpha_{s t r}}+\Delta_{i}\left(M_{U}\right) .
$$

The above relation looks very similar to the well-known unification condition in Supersymmetric Grand Unified Theories (SuSy-GUTs) where the unification scale is about $M_{U} \sim 10^{16} \mathrm{GeV}$ and $\Delta_{i}\left(M_{U}\right)=0$ in the $\overline{D R}$ renormalization scheme; in SuSy-GUTs the normalization constants $k_{i}$ are fixed only for the gauge couplings $\left(k_{1}=k_{2}=k_{3}=1\right.$,

$k_{e m}=\frac{3}{8}$ ), but there are no relations among gauge and Yukawa couplings at all. In string effective theories, however, the normalization constants $\left(k_{i}\right)$ are known for both gauge and Yukawa interactions. Furthermore, $\Delta_{i}\left(M_{U}\right)$ are calculable finite quantities for any particular string solution. Thus, the predictability of a given string solution is extended for all low energy coupling constants $\alpha_{i}\left(M_{Z}\right)$ once the string-induced corrections $\Delta_{i}\left(M_{U}\right)$ are determined.

It turns out that $\Delta_{i}\left(M_{U}\right)$ are non-trivial functions of the vacuum expectation values of some gauge singlet fields [9, [10, [1], 16], $\left\langle T_{A}\right\rangle=t_{A}$, the so-called moduli (the moduli fields are flat directions at the string classical level and they remain flat in string perturbation theory, in the exact supersymmetry limit). The $\Delta_{i}\left(t_{A}\right)$ are target space duality invariant functions, which depend on the particular string ground state. $\beta$-functions in string theory were calculated first in [17]. Several results for $\Delta_{i}$ exist by now [9, 10, 11, 16] in the exact supersymmetric limit in many string solutions based on orbifold [2] and fermionic constructions [5]. As we will see later $\Delta_{i}$ are, in principle, well defined calculable quantities once we perform our calculations at the string level where all interactions including gravity are consistently defined. Although at this stage we do not know the details of supersymmetry breaking we should stress here that for dimensionless couplings in four dimensions choosing the IR (low energy) scale above the threshold of supersymmetric partners the one-loop corrections to these couplings is exact because soft breaking terms do not affect such renormalizations. For soft breaking parameters only qualitative results exist up to 
now although this is a subject of intensive study.

The main obstruction in determining the exact form of the string radiative corrections $\Delta_{i}\left(M_{U}\right)$ is related to the infrared divergences of on-shell calculations in string theory. In field theory, we can avoid this problem using off-shell calculations. In first quantized string theory we cannot do that since we do not know how to go off-shell. Even in field theory there are problems in defining an infrared regulator for chiral fermions especially in the presence of spacetime supersymmetry.

The idea is to modify slightly the ground state of interest in string theory so that it develops a mass gap. It is known already in field theory that a space of negative curvature provides bosonic fields (scalars, vectors etc.) with such a mass gap. The case of fermions (especially chiral ones) though is more subtle. We will see however that string theory contains the fields (namely the antisymmetric tensor) which when they acquire some suitable expectation values they can provide a mass gap for (chiral) fermions.

Let us indicate here how an expectation value for the dilaton can give masses to bosonic fields. The dilaton couples generically to (massless) bosonic fields in a universal fashion:

$$
S_{T}=\int e^{-2 \Phi} \partial_{\mu} T \partial^{\mu} T
$$

where we considered the case of a scalar field $T$. To find the spectrum of the fluctuations of $\mathrm{T}$ we have to define $\tilde{T}=e^{-\Phi} T$ so that the action becomes

$$
S_{T}=\int \partial_{\mu} \tilde{T} \partial^{\mu} \tilde{T}+\left[\partial_{\mu} \Phi \partial^{\mu} \Phi-\partial_{\mu} \partial^{\mu} \Phi\right] \tilde{T}^{2}
$$

It is obvious that if $\langle\Phi\rangle=Q_{\mu} x^{\mu}$ then the scalar $\tilde{T}$ acquires a mass ${ }^{2} Q_{\mu} Q^{\mu}$ which is

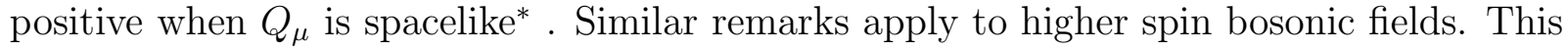
mechanism via the dilaton cannot give masses to fermions since the extra shift obtained by the redefinition is a total divergence.

Consider a chiral fermion with its universal coupling to the antisymmetric tensor:

$$
S_{\psi}=\int \bar{\psi}[\stackrel{\leftrightarrow}{\partial}+\text { H] } \psi
$$

where $H_{\mu}=\epsilon_{\mu}{ }^{\nu \rho \sigma} H_{\nu \rho \sigma}$ is th dual of the field stregth of the antisymmetric tensor. If $\left\langle H_{\mu}\right\rangle=Q_{\mu}$ then the Dirac operator acquires a mass gap proportional to $Q_{\mu} Q^{\mu}$.

Thus we need to find exact string ground states (CFTs) which implement the mechanism sketched above.

In particular we would like our background to have the following properties:

1. The string spectrum must have a mass gap $\mu^{2}$. In particular, chiral fermions should be regulated consistently.

2. We should be able to take the limit $\mu^{2} \rightarrow 0$.

3. It should have $c=(6,4)$ so that it can be coupled to any internal CFT with $c=(9,22)$.

\footnotetext{
*This was observed in [18] with $Q_{\mu}$ being timelike.
} 
4. It should preserve as many spacetime supersymmetries of the original theory, as possible.

5. We should be able to calculate the regulated quantities relevant for the effective field theory.

6. Vertices for spacetime fields (like $F_{\mu \nu}^{a}$ ) should be well defined operators on the world-sheet.

7. The theory should be modular invariant (which guarantees the absence of anomalies).

8. Such a regularization should be possible also at the effective field theory level. In this way, calculations in the fundamental theory can be matched without any ambiguity to those of the effective field theory.

CFTs with the properties above employ special four-dimensional spaces as superconformal building blocks with $\hat{c}=4$ and $N=4$ superconformal symmetry [12, 14. The full spectrum of string excitations for the superstring solutions based on those four-dimensional subspaces, can be derived using the techniques developed in [14]. The spectrum does have a mass gap, which is proportional to the curvature of the non-trivial four-dimensional spacetime. Comparing the spectrum in a flat background with that in curved space we observe a shifting of all massless states by an amount proportional to the spacetime curvature, $\Delta m^{2}=Q^{2}=\mu^{2}$, where $Q$ is the Liouville background charge and $\mu$ is the IR cutoff. What is also interesting is that the shifted spectrum in curved space is equal for bosons and fermions due to the existence of a new space-time supersymmetry defined in curved spacetime [12, 14]. Therefore, our curved spacetime infrared regularization is consistent with supersymmetry and can be used either in field theory or string theory.

Most of the work presented here has already appeared in [19, 20] We present also some new results in section 4 .

\section{The IR regulated String Theory}

We will choose the 4-D CFT which will replace flat space to be the $S U(2)_{k} \otimes R_{Q}$ model. It contains a non-compact direction with a linear dilaton $\Phi=Q x^{0}$ as well as the $S U(2)_{k}$ WZW model. Q is related to $k$ as $Q=1 / \sqrt{k+2}$ so that the CFT has the same central charge as flat space. We will define $\mu^{2}=1 /(k+2), \mu$ is directly related to the mass gap of the regulated theory. The GSO projection couples the $\mathrm{SU}(2)$ spin with the spacetime helicity [19]. This effectively projects out the half-integral spins and replaces $S U(2)$ with $S O(3) . k$ should be an even positive integer for consistency. For any ground state of the heterotic string with $N<4$ spacetime supersymmetry the regulated vaccum amplitude turns out to be

$$
Z(\mu)=\frac{1}{V(\mu)} \Gamma_{0}(\mu) Z_{0}
$$


where $V(\mu)=1 / 8 \pi \mu^{3}$ is the volume of the nontrivial background and $Z_{0}$ is the vacuum amplitude for the unregulated theory, which can be written as

$$
Z_{0}(\tau, \bar{\tau})=\frac{1}{\operatorname{Im} \tau|\eta|^{4}} \sum_{a, b=0}^{1} \frac{\theta\left[\begin{array}{l}
a \\
b
\end{array}\right]}{\eta} C\left[\begin{array}{l}
a \\
b
\end{array}\right](\tau, \bar{\tau})
$$

where we have separated the generic 4-d contribution. The factor $C\left[\begin{array}{l}a \\ b\end{array}\right]$ is the Trace in the $\left(\begin{array}{l}a \\ b\end{array}\right)$ sector of the internal CFT. Finally, $\Gamma_{0}(\mu)$ is proportional to the $S O(3)_{k}$ partition function

$$
\Gamma_{0}(\mu)=\frac{1}{2}\left[(\operatorname{Im} \tau)^{\frac{1}{2}} \eta(\tau) \bar{\eta}(\bar{\tau})\right]^{3} \sum_{a, b=0}^{1} e^{-i \pi k a b / 2} \sum_{l=0}^{k} e^{i \pi b l} \chi_{l}(\tau) \bar{\chi}_{(1-2 a) l+a k}(\bar{\tau})
$$

where $\chi_{l}$ are the standard $S U(2)_{k}$ characters. We have also the correct limit $Z(\mu) \rightarrow Z_{0}$ as $\mu \rightarrow 0$.

There is a simple expression for $\Gamma_{0}(\mu)$

$$
\Gamma_{0}(\mu)=-\frac{1}{2 \pi} X^{\prime}(\mu)
$$

where prime stands for derivative with respect to $\mu^{2}$ and

$$
X(\mu)=\frac{1}{2 \mu} \sum_{m, n \in Z} e^{i \pi(m+n+m n)} \exp \left[-\frac{\pi}{4 \mu^{2} \operatorname{Im} \tau}|m-n \tau|^{2}\right]=\sqrt{\operatorname{Im} \tau} \sum_{m, n \in Z} e^{i \pi n} q^{\frac{1}{4} Q_{L}^{2}} \bar{q}^{\frac{1}{4} Q_{R}^{2}}
$$

with

$$
Q_{L}=2 \mu\left(m-\frac{n+1}{2}\right)+\frac{n}{2 \mu}, Q_{R}=2 \mu\left(m-\frac{n+1}{2}\right)-\frac{n}{2 \mu}
$$

It can be also written in terms of the usual torroidal sum:

$$
\begin{gathered}
X(\mu)=Z_{T}(\mu)-Z_{T}(2 \mu) \\
Z_{T}(\mu)=Z_{T}(1 / \mu)=\sqrt{\operatorname{Im} \tau} \sum_{m, n \in Z} q^{\frac{1}{4}(m \mu+n / \mu)^{2}} \bar{q}^{\frac{1}{4}(m \mu-n / \mu)^{2}}
\end{gathered}
$$

Note that $X(\mu)$ is modular invariant.

The leading infrared behaviour can be read from (2.4), (2.5) to be

$$
Z(\mu) \rightarrow \sqrt{\operatorname{Im} \tau} e^{-\pi \operatorname{Im} \tau \mu^{2}}
$$

as $\operatorname{Im} \tau \rightarrow \infty$ that indicates explicitly the presence of the mass gap.

More details on this theory can be found in [19, 20].

\section{$3 \quad$ Non-zero $\mathbf{F}_{\mu \nu}^{\mathrm{a}}$ and $R_{\mu \nu}^{\rho \sigma}$ Background in Superstrings}

As mentioned in the introduction in order to calculate the renormalization of the effective couplings we need to turn on backgrounds for gauge and gravitational fields. Thus our aim is to define the deformation of the two-dimensional superconformal theory which 
corresponds to a non-zero field strength $F_{\mu \nu}^{a}$ and $R_{\mu \nu \rho \sigma}$ background and find the integrated one-loop partition function $Z(\mu, F, \mathcal{R})$, where $F$ is by the magnitude of the field strength, $F^{2} \equiv\left\langle F_{\mu \nu}^{a} F_{a}^{\mu \nu}\right\rangle$ and $\mathcal{R}$ is that of the curvature, $\left\langle R_{\mu \nu \rho \sigma} R^{\mu \nu \rho \sigma}\right\rangle=\mathcal{R}^{2}$.

$$
Z\left[\mu, F_{i}, \mathcal{R}\right]=\frac{1}{V(\mu)} \int_{\mathcal{F}} \frac{d \tau d \bar{\tau}}{(\operatorname{Im} \tau)^{2}} Z\left[\mu, F_{i}, \mathcal{R} ; \tau, \bar{\tau}\right]
$$

The index $i$ labels different simple or $U(1)$ factors of the gauge group of the ground state.

In flat space, a small non-zero $F_{\mu \nu}^{a}$ background gives rise to an infinitesimal deformation of the 2-d $\sigma$-model action given by,

$$
\Delta S^{2 d}\left(F^{(4)}\right)=\int d z d \bar{z} F_{\mu \nu}^{a}\left[x^{\mu} \partial_{z} x^{\nu}+\psi^{\mu} \psi^{\nu}\right] \bar{J}_{a}
$$

Observe that for $F_{\mu \nu}^{a}$ constant (constant magnetic field), the left moving operator $\left[x^{\mu} \partial_{z} x^{\nu}+\right.$ $\left.\psi^{\mu} \psi^{\nu}\right]$ is not a well-defined $(1,0)$ operator on the world sheet. Even though the right moving Kac-Moody current $\bar{J}_{a}$ is a well-defined $(0,1)$ operator, the total deformation is not integrable in flat space. Indeed, the 2 -d $\sigma$-model $\beta$-functions are not satisfied in the presence of a constant magnetic field. This follows from the fact that there is a non-trivial back-reaction on the gravitational background due the non-zero magnetic field.

The important property of our non-trivial spacetime background is that we can solve exactly for the back-reaction. First observe that the deformation that corresponds to a constant magnetic field $B_{i}^{a}=\epsilon_{o i j k} F_{a}^{i k}$ is a well-defined $(1,1)$ integrable deformation, which breaks the $(2,2)$ superconformal invariance but preserves the $(1,0)$ world-sheet supersymmetry:

$$
\Delta S^{2 d}\left(W_{k}^{(4)}\right)=\int d z d \bar{z} B_{i}^{a}\left[I^{i}+\frac{1}{2} \epsilon^{i j k} \psi_{j} \psi_{k}\right] \bar{J}_{a}
$$

where $I^{i}$ is anyone of the $S U(2)_{k}$ currents. The deformed partition function is not zero due to the breaking of $(2,2)$ supersymmetry. It can be shown that this is the correct replacement of the Lorentz current in the flat case [19]. More details on the physics of such magnetic fields can be found in [20].

The $\mathcal{R}$ perturbation is

$$
\Delta S(\mathcal{R})=\int d z d \bar{z} \mathcal{R}\left[I^{3}+\psi^{1} \psi^{2}\right] \bar{I}^{3}
$$

This turns on a Riemann tensor with constant scalar curvature equal to $6 \mathcal{R}$ as well as an antisymmetric tensor and dilaton.

Due to the rotation invariance in $S^{3}$ we can choose $B_{i}^{a}=F \delta_{i}^{3}$ without loss of generality. The vector $B_{i}^{a}$ indicates the direction in the gauge group space of the right-moving affine currents.

The moduli space of the $F$ deformation is then given by the $S O(1, n) / S O(n)$ Lorentzianlattice boosts with $n$ being the rank of the right-moving gauge group. We therefore conclude that the desired partition function $Z\left(\mu, F_{i}, \mathcal{R}=0\right)$ is given in terms of the moduli of

${ }^{*}$ Magnetic backgrounds in closed string theory have been also discussed in [21, 22]. 
the $\Gamma(1, n)$ lorentzian lattice. The constant gravitational background $R_{k l}^{i j}=\mathcal{R} \epsilon^{3 i j} \epsilon_{3 k l}$ can also be included exactly by an extra boost, in which case the lattice becomes $\Gamma(1, n+1)$.

Let us denote by $\mathcal{Q}$ the fermionic lattice momenta associated to the left-moving $U(1)$ current $\partial H=\psi^{1} \psi^{2}$, by $J^{3}, \bar{J}^{3}$ the zero mode of the respective (left or right) $S U(2)_{k}$ current, by $\overline{\mathcal{Q}}_{i}$ the zero mode of a Cartan generator of the left moving current algebra generating a simple or $\mathrm{U}(1)$ component of the gauge group. The currents are normalized so that $J_{i}^{a}(z) J_{j}^{b}(0)=k_{i} \delta_{i j} \delta^{a b} / 2 z^{2}+\ldots$. Note that this fixes the normalization of the Casimirs.

In terms of these charges the undeformed partition function can be written as

$$
\operatorname{Tr}\left[\exp \left[-2 \pi \operatorname{Im} \tau\left(\mathrm{L}_{0}+\overline{\mathrm{L}}_{0}\right)+2 \pi \mathrm{iRe} \tau\left(\mathrm{L}_{0}-\overline{\mathrm{L}}_{0}\right)\right]\right]
$$

where

$$
\mathrm{L}_{0}=\frac{1}{2} \mathcal{Q}^{2}+\frac{\left(J^{3}\right)^{2}}{k}+\cdots, \overline{\mathrm{L}}_{0}=\sum_{i} \frac{\overline{\mathcal{Q}}_{i}^{2}}{k_{i}}+\frac{\left(\bar{J}^{3}\right)^{2}}{k}+\cdots
$$

where the dots stand for operators that do not involve $J^{3}, \bar{J}^{3}, \mathcal{Q}, \overline{\mathcal{Q}}_{i}$.

The $(1,1)$ perturbation that turns on constant gauge field strengths $F_{i}, i=1,2, \ldots, n$ as well as a constant curvature $\mathcal{R}$ background produces an $\mathrm{O}(1,1+\mathrm{n})$ 2-parameter boost in $O(2,2)$, acting on the charge lattice above.

$$
\begin{gathered}
\delta L_{0}=\delta \bar{L}_{0}=\left(\mathcal{Q}+J^{3}\right)\left(\mathcal{R} \bar{J}^{3}+F_{i} \bar{Q}_{i}\right)+ \\
+\frac{-1+\sqrt{1+(k+2)\left(k_{i} F_{i}^{2}+k \mathcal{R}^{2}\right)}}{2}\left[\frac{\left(\mathcal{Q}+J^{3}\right)^{2}}{k+2}+\frac{\left(F_{i} \bar{Q}_{i}+\mathcal{R} \bar{J}^{3}\right)^{2}}{k_{i} F_{i}^{2}+k \mathcal{R}^{2}}\right]
\end{gathered}
$$

The first term is the standard perturbation while the second term is the back-reaction necessary for conformal and modular invariance. Expanding the partition function in a power series in $F_{i}, \mathcal{R}$

$$
Z(\mu, F, \mathcal{R})=\sum_{n_{i}, m=0}^{\infty} \prod_{i=1}^{n} F_{i}^{n_{i}} \mathcal{R}^{m} Z_{n_{i}, m}(\mu)
$$

we can extract the integrated correlators $Z_{n_{i}, m}=\left\langle\prod_{i=1}^{n} F_{i}^{n_{i}} R^{m}\right\rangle$.

$$
\begin{gathered}
\left\langle F_{i}\right\rangle=-4 \pi \operatorname{Im} \tau\left\langle\left(\mathcal{Q}+J^{3}\right)\right\rangle\left\langle\bar{Q}_{i}\right\rangle \\
\langle\mathcal{R}\rangle=-4 \pi \operatorname{Im} \tau\left\langle\left(\mathcal{Q}+J^{3}\right)\right\rangle\left\langle\bar{J}^{3}\right\rangle \\
\left\langle F_{i}^{2}\right\rangle=8 \pi^{2} \operatorname{Im} \tau^{2}\left[\left\langle\left(\mathcal{Q}+J^{3}\right)^{2}\right\rangle-\frac{(k+2)}{8 \pi \operatorname{Im} \tau}\right]\left[\left\langle\left(\bar{Q}_{i}\right)^{2}\right\rangle-\frac{k_{i}}{8 \pi \operatorname{Im} \tau}\right]-\frac{k_{i}(k+2)}{8} \\
\left\langle\mathcal{R}^{2}\right\rangle=8 \pi^{2} \operatorname{Im} \tau^{2}\left[\left\langle\left(\mathcal{Q}+J^{3}\right)^{2}\right\rangle-\frac{k+2}{8 \pi \operatorname{Im} \tau}\right]\left[\left\langle\left(\bar{J}^{3}\right)^{2}\right\rangle-\frac{k}{8 \pi \operatorname{Im} \tau}\right]-\frac{k(k+2)}{8} \\
\left\langle\mathcal{R} F_{i}\right\rangle=16 \pi^{2} \operatorname{Im} \tau^{2}\left\langle\bar{J}^{3} \bar{Q}_{i}\right\rangle\left[\left\langle\left(\mathcal{Q}+J^{3}\right)^{2}\right\rangle-\frac{k+2}{8 \pi \operatorname{Im} \tau}\right] \\
\left\langle F_{i} F_{j}\right\rangle=16 \pi^{2} \operatorname{Im} \tau^{2}\left\langle\bar{Q}_{i} \bar{Q}_{j}\right\rangle\left[\left\langle\left(\mathcal{Q}+J^{3}\right)^{2}\right\rangle-\frac{k+2}{8 \pi \operatorname{Im} \tau}\right]
\end{gathered}
$$

where we should always remember that $k+2=1 / \mu^{2}$. 
For Supersymmetric ground states we have simplifications

$$
\begin{aligned}
& \left\langle F_{i}^{2}\right\rangle_{S U S Y}=8 \pi^{2} \operatorname{Im} \tau^{2}\left\langle\mathcal{Q}^{2}\right\rangle\left[\left\langle\left(\bar{Q}_{i}\right)^{2}\right\rangle-\frac{k_{i}}{8 \pi \operatorname{Im} \tau}\right] \\
& \left\langle\mathcal{R}^{2}\right\rangle_{S U S Y}=8 \pi^{2} \operatorname{Im} \tau^{2}\left\langle\mathcal{Q}^{2}\right\rangle\left[\left\langle\left(\bar{J}^{3}\right)^{2}\right\rangle-\frac{k}{8 \pi \operatorname{Im} \tau}\right]
\end{aligned}
$$

Renormalizations of higher terms can be easily computed. We give here the expression for an $F_{i}^{4}$ term,

$$
\begin{gathered}
\left\langle F_{i}^{4}\right\rangle=\frac{(4 \pi \operatorname{Im} \tau)^{4}}{24}\left\langle\left[\left(\mathcal{Q}+J^{3}\right)^{4}\left(\bar{Q}_{i}\right)^{4}-\frac{3}{4 \pi \operatorname{Im} \tau}\left(\mathcal{Q}+J^{3}\right)^{2}\left(\bar{Q}_{i}\right)^{2}\left(\left(k_{i}\left(\mathcal{Q}+J^{3}\right)^{2}+\right.\right.\right.\right. \\
\left.+(k+2)\left(\bar{Q}_{i}\right)^{2}\right)+\frac{3}{4(4 \pi \operatorname{Im} \tau)^{2}}\left[k_{i}\left(\mathcal{Q}+J^{3}\right)^{2}+(k+2)\left(\bar{Q}_{i}\right)^{2}\right]^{2}- \\
\left.-\frac{3 k_{i}(k+2)}{2(4 \pi \operatorname{Im} \tau)^{3}}\left[\left[k_{i}\left(\mathcal{Q}+J^{3}\right)^{2}+(k+2)\left(\bar{Q}_{i}\right)^{2}\right]\right]\right\rangle
\end{gathered}
$$

The charge $\mathcal{Q}$ in the above formulae acts on the helicity $\vartheta$-function $\vartheta\left[\begin{array}{l}\alpha \\ \beta\end{array}\right](\tau, v)$ as differentiation with respect to $v$ divide by $2 \pi i$. The charges $\bar{Q}_{i}$ act also as $v$ derivatives on the respective characters of the current algebra. $J^{3}, \bar{J}^{3}$ act in the level- $k \vartheta$-function present in $S O(3)_{k}$ partition function (due to the parafermionic decomposition).

\section{One-loop Corrections to the Coupling Constants}

We now focus on the one-loop correction to the gauge couplings. Bearing anomalous $\mathrm{U}(1)$ 's we can immediately see from (3.9) that $\left\langle F_{i}\right\rangle=0$ and $\left\langle F_{i} F_{j}\right\rangle=0$ for $i \neq j$. The conventionally normalized one-loop correction is

$$
\left.\frac{16 \pi^{2}}{g_{i}^{2}}\right|_{1-\text { loop }}=-\frac{1}{(2 \pi)^{2}} \int_{\mathcal{F}} \frac{d^{2} \tau}{\operatorname{Im} \tau^{2}}\left\langle F_{i}^{2}\right\rangle
$$

Putting everything together we obtain

$$
\begin{gathered}
\left.\frac{16 \pi^{2}}{g_{i}^{2}}\right|_{1-\text { loop }}=-\frac{i}{\pi^{2} V(\mu)} \int_{\mathcal{F}} \frac{d^{2} \tau}{\operatorname{Im} \tau|\eta|^{4}} \sum_{a, b=0}^{1}\left[X^{\prime}(\mu) \partial_{\tau}\left(\frac{\theta\left[\begin{array}{l}
a \\
b
\end{array}\right]}{\eta}\right)+\frac{1}{6 \mu^{2}} \dot{X}^{\prime}(\mu) \frac{\theta\left[\begin{array}{l}
a \\
b
\end{array}\right]}{\eta}\right] \times \\
\times \operatorname{Tr}_{a, b}^{I}\left[\left\langle\left(\bar{J}^{i}\right)^{2}\right\rangle-\frac{k_{i}}{8 \pi \operatorname{Im} \tau}\right]--\frac{k_{i}}{64 \pi^{3} \mu^{2} V(\mu)} \int_{\mathcal{F}} \frac{d^{2} \tau}{\operatorname{Im} \tau^{2}} X^{\prime}(\mu) Z_{0}
\end{gathered}
$$

where dot stands for derivative with respect to $\tau$ and $\operatorname{Tr}_{a b}^{I}$ stands for the trace in the $\left(\begin{array}{l}a \\ b\end{array}\right)$ sector of the internal CFT. Eq. (4.2) is valid also for non-supersymmetric ground states. 
When we have $N \geq 1$ supersymmetry it simplifies to

$$
\left.\frac{16 \pi^{2}}{g_{i}^{2}}\right|_{1-\text { loop }} ^{S U S Y}=-\frac{i}{\pi^{2} V(\mu)} \int_{\mathcal{F}} \frac{d^{2} \tau}{\operatorname{Im} \tau|\eta|^{4}} \sum_{a, b=0}^{1}\left[X^{\prime}(\mu) \frac{\partial_{\tau} \theta\left[\begin{array}{l}
a \\
b
\end{array}\right]}{\eta}\right] \operatorname{Tr}_{a, b}^{I}\left[\left\langle\left(\bar{J}^{i}\right)^{2}\right\rangle-\frac{k_{i}}{8 \pi \operatorname{Im} \tau}\right]
$$

The general formula (4.2) can be split in the following way

$$
\begin{gathered}
\left.\frac{16 \pi^{2}}{g_{i}^{2}}\right|_{1-\text { loop }}=I_{1}+I_{2}+I_{3} \\
I_{1}=-\frac{i}{\pi^{2} V(\mu)} \int_{\mathcal{F}} \frac{d^{2} \tau}{\operatorname{Im} \tau|\eta|^{4}} X^{\prime}(\mu) \sum_{a, b=0}^{1} \partial_{\tau}\left(\frac{\theta[a]}{\eta}\right) \operatorname{Tr}_{a, b}^{I}\left[\left\langle\left(\bar{J}^{i}\right)^{2}\right\rangle-\frac{k_{i}}{8 \pi \operatorname{Im} \tau}\right] \\
I_{2}=-\frac{i}{6 \pi^{2} \mu^{2} V(\mu)} \int_{\mathcal{F}} \frac{d^{2} \tau}{\operatorname{Im} \tau|\eta|^{4}} \dot{X}^{\prime}(\mu) \sum_{a, b}^{1} \frac{\theta\left[\begin{array}{l}
a \\
l
\end{array}\right]}{\eta} \operatorname{Tr}_{a, b}^{I}\left[\left\langle\left(\bar{J}^{i}\right)^{2}\right\rangle-\frac{k_{i}}{8 \pi \operatorname{Im} \tau}\right] \\
I_{3}=-\frac{k_{i}}{64 \pi^{3} \mu^{2} V(\mu)} \int_{\mathcal{F}} \frac{d^{2} \tau}{\operatorname{Im} \tau^{2}} X^{\prime}(\mu) Z_{0}
\end{gathered}
$$

All the integrands are separately modular invariant. The universal term in $I_{1}$ is due to an axion tadpole. $I_{3}$ is the contribution of a dilaton tadpole. $I_{2}$ are extra helicity contributions due to the curved background. Moreover $I_{2}, I_{3}$ have power IR divergences which reflect quadratic divergences in the effective field theory. $I_{2}, I_{3}$ are zero for supersymmetric ground states.

We will now analyse the contribution of the massless sector to the one-loop corrections. Since

$$
-\frac{1}{i \pi} \partial_{\tau}\left(\frac{\theta[a]}{\eta}\right) \rightarrow(-1)^{F}\left(\frac{1}{12}-\chi^{2}\right)
$$

where $\chi$ is the helicity of a state, we obtain

$$
\begin{gathered}
I_{1}^{\text {massless }}=-\frac{1}{\pi} \operatorname{Str}\left[Q_{i}^{2}\left(\frac{1}{12}-\chi^{2}\right)\right] \mathbf{J}_{1}(\mu)+\frac{k_{i}}{8 \pi^{2}} \operatorname{Str}\left[\frac{1}{12}-\chi^{2}\right] \mathbf{J}_{2}(\mu) \\
I_{2}^{\text {massless }}=-\frac{1}{12 \pi^{2} \mu^{2}} \operatorname{Str}\left[Q_{i}^{2}\right] \mathbf{J}_{2}(\mu)+\frac{k_{i}}{48 \pi^{3} \mu^{2}} \operatorname{Str}[\mathbf{1}] \mathbf{J}_{3}(\mu) \\
I_{3}^{\text {massless }}=-\frac{k_{i}}{64 \pi^{3} \mu^{2}} \operatorname{Str}[\mathbf{1}] \mathbf{J}_{3}(\mu)
\end{gathered}
$$

Here

$$
\mathbf{J}_{n} \equiv \frac{1}{V(\mu)} \int_{\mathcal{F}} \frac{d^{2} \tau}{\operatorname{Im} \tau^{n}} X^{\prime}(\mu)
$$

which can be evaluated to be

$$
\begin{gathered}
\mathbf{J}_{1}(\mu)=2 \pi \log \mu^{2}+2 \pi\left(\log \pi+\gamma_{E}-3+\frac{3}{2} \log 3\right)+\mathcal{O}\left(e^{-\frac{1}{\mu^{2}}}\right) \\
\mathbf{J}_{2}(\mu)=-\frac{4 \pi^{2}}{3}\left(1+\mu^{2}\right), \quad \mathbf{J}_{3}(\mu)=-\pi \log 3-\frac{28 \pi^{3}}{15} \mu^{4}+\mathcal{O}\left(e^{-\frac{1}{\mu^{2}}}\right)
\end{gathered}
$$


We would like now to describe the same calculation in the effective field theory.

This calculation proceeds along the same lines as above taking into account the following differences.

- Now $\mu_{e}^{2}=1 / k$ and $V\left(\mu_{e}\right)=1 /\left(8 \pi \mu_{e}^{3}\right)$.

- $\Gamma_{0} / V\left(\mu_{e}\right)$ is given by the momentum mode part of the stringy expression:

$$
\frac{\Gamma_{0}}{V\left(\mu_{e}\right)}=-4 \mu_{e}^{3} \partial_{\mu_{e}^{2}} \sqrt{\operatorname{Im} \tau} \sum_{n \in Z} e^{-\pi \operatorname{Im} \tau \mu_{e}^{2}(2 n+1)^{2}}
$$

- There is an incomplete cancelation of the $1 / 8 \pi \mu^{2} \operatorname{Im} \tau$ piece in (3.9c). What remains is $1 / 4 \pi \operatorname{Im} \tau$.

- The integral over $\operatorname{Im} \tau$ is done from 0 to $\infty$. We will regulate the ultraviolet divergences by the Schwinger regularization which amounts to integrating the parameter $\mathrm{t}$ in the interval $\left[1 / \pi \Lambda^{2}, \infty\right]$.

Then,

$$
\left.\frac{16 \pi^{2}}{g_{i}^{2}}\right|_{1-l o o p} ^{E F T}=L_{1}+L_{2}+L_{3}
$$

where

$$
\begin{gathered}
L_{1}=-\frac{1}{\pi} \operatorname{Str}\left[Q_{i}^{2}\left(\frac{1}{12}-\chi^{2}\right)\right] \mathbf{K}_{1}\left(\mu_{e}\right)+\frac{k_{i}}{8 \pi^{2}} \operatorname{Str}\left[\frac{1}{12}-\chi^{2}\right] \mathbf{K}_{2}\left(\mu_{e}\right) \\
L_{2}=-\frac{1}{4 \pi^{2}}\left(1+\frac{1}{3 \mu_{e}^{2}}\right) \operatorname{Str}\left[Q_{i}^{2}\right] \mathbf{K}_{2}\left(\mu_{e}\right)+\frac{k_{i}}{16 \pi^{3}}\left(\frac{1}{2}+\frac{1}{3 \mu_{e}^{2}}\right) \operatorname{Str}[\mathbf{1}] \mathbf{K}_{3}\left(\mu_{e}\right) \\
L_{3}=-\frac{k_{i}\left(1+2 \mu_{e}^{2}\right)}{64 \pi^{3} \mu_{e}^{2}} \operatorname{Str}[\mathbf{1}] \mathbf{K}_{3}\left(\mu_{e}\right)
\end{gathered}
$$

and

$$
\mathbf{K}_{n}\left(\mu_{e}\right) \equiv \frac{1}{V\left(\mu_{e}\right)} \int_{\frac{1}{\pi \Lambda^{2}}}^{\infty} \frac{d t}{t^{n}} \partial_{\mu_{e}^{2}} \sqrt{t}\left[\theta_{3}\left(i t \mu_{e}^{2}\right)-\theta_{3}\left(4 i t \mu_{e}^{2}\right)\right]
$$

The integrals can again be evaluated

$$
\mathbf{K}_{1}\left(\mu_{e}, \Lambda\right)=4 \pi \log \left(\mu_{e} / \Lambda\right)+2 \pi\left(\gamma_{E}-2\right)+\mathcal{O}\left(e^{-\Lambda^{2} / \mu_{e}^{2}}\right)
$$

and for $n>1$

$\mathbf{K}_{n}\left(\mu_{e}, \Lambda\right)=-\frac{2 \pi}{n-1} \Lambda^{2 n-2}+8 \pi^{2-n}(2 n-3)\left(1-2^{2 n-3}\right) \Gamma(n-1) \zeta(2 n-2) \mu_{e}^{2 n-2}+\mathcal{O}\left(e^{-\Lambda^{2} / \mu_{e}^{2}}\right)$

In a similar fashion we can calculate the string one-loop correction to the $R^{2}$ coupling with the result

$$
\left.\frac{1}{g_{R^{2}}^{2}}\right|_{1-\text { loop }}=\frac{4}{\pi V(\mu)} \int_{\mathcal{F}} \frac{d^{2} \tau}{\operatorname{Im} \tau|\eta|^{4}} \sum_{a, b}^{1}\left[\partial_{\tau}\left(\frac{\theta\left[\begin{array}{l}
a \\
b
\end{array}\right]}{\eta}\right)\left(\bar{G}_{2}-\frac{1}{6 \mu^{2}} \partial_{\bar{\tau}}\right) X^{\prime}\right.
$$




$$
\left.+\frac{1}{6 \mu^{2}} \frac{\theta\left[\begin{array}{c}
a \\
b
\end{array}\right]}{\eta}\left(\bar{G}_{2}-\frac{1}{6 \mu^{2}} \partial_{\bar{\tau}}\right) \partial_{\tau} X^{\prime}\right]+\frac{k(k+2)}{16 \pi V(\mu)} \int_{\mathcal{F}} \frac{d^{2} \tau}{\operatorname{Im} \tau^{2}} X^{\prime} Z_{0}
$$

where

$$
\bar{G}_{2} \equiv \partial_{\bar{\tau}} \log \bar{\eta}+\frac{i}{4 \operatorname{Im} \tau}=\frac{1}{2} \partial_{\bar{\tau}} \log \left[\operatorname{Im} \tau \bar{\eta}^{2}\right]
$$

One-loop corrections to higher dimension operators can also be computed. We give here the result for $F_{\mu \nu}^{4}$ for $Z_{2} \times Z_{2}$ orbifold compactifications of the heterotic string. This correction gets contributions from all sectors including $N=4$ ones and it is thus interesting for studying decompactification problems in string theory. The $N=4$ sector contribution to the $F_{\mu \nu}^{4}$ term for the $E_{8}$ gauge group can be computed from (3.12) to be

$$
\begin{gathered}
\left.\frac{1}{g_{F^{4}}^{2}}\right|_{1-\text { loop }} ^{E_{8}}=\frac{1}{V(\mu)} \int_{\mathcal{F}} \frac{d^{2} \tau}{\operatorname{Im} \tau^{2}} X^{\prime}(\mu) \prod_{i=1}^{3}\left[\operatorname{Im} \tau \Gamma_{2,2}\left(T_{i}, U_{i}\right)\right] \sum_{a, b=0}^{1} \frac{\bar{\vartheta}^{8}\left[\begin{array}{l}
a \\
b
\end{array}\right]}{\bar{\eta}^{24}} \times \\
\times \sum_{\gamma, \delta=0}^{1} \bar{\vartheta}^{7}\left[\begin{array}{l}
\gamma \\
\delta
\end{array}\right]\left(\frac{i}{\pi} \partial_{\bar{\tau}}-\frac{5}{2 \pi \operatorname{Im} \tau}\right)\left(\frac{i}{\pi} \partial_{\bar{\tau}}-\frac{1}{4 \pi \operatorname{Im} \tau}\right) \bar{\vartheta}\left[\begin{array}{l}
\gamma \\
\delta
\end{array}\right]
\end{gathered}
$$

\section{$5 \quad$ IR Flow Equations for Couplings}

Once we have obtained the one-loop corrections to the coupling constants we can observe that they satisfy scaling type flows. We will present here IR Flow Equations (IRFE) for differences of gauge couplings.

The existence of IRFE is due to differential equations satisfied by the lattice sum of an arbitrary (d,d) lattice,

$$
Z_{d, d}=\operatorname{Im} \tau^{d / 2} \sum_{P_{L}, P_{R}} e^{i \pi \tau P_{L}^{2} / 2-i \pi \bar{\tau} P_{R}^{2} / 2}
$$

where

$$
P_{L, R}^{2}=\vec{n} G^{-1} \vec{n}+2 \vec{m} B G^{-1} \vec{n}+\vec{m}\left[G-B G^{-1} B\right] \vec{m} \pm 2 \vec{m} \cdot \vec{n}
$$

$\vec{m}, \vec{n}$ are integer d-dimesional vectors and $G_{i j}\left(B_{i j}\right)$ is a real symmetric (antisymmetric) matrix. $Z_{d, d}$ is $O(d, d, Z)$ and modular invariant. Moreover it satisfies the following second order differential equation円:

$$
\left[\left(G_{i j} \frac{\partial}{\partial G_{i j}}+\frac{1-d}{2}\right)^{2}+2 G_{i k} G_{j l} \frac{\partial^{2}}{\partial B_{i j} \partial B_{k l}}-\frac{1}{4}-4 \operatorname{Im} \tau^{2} \frac{\partial^{2}}{\partial \tau \partial \bar{\tau}}\right] Z_{d, d}=0
$$

The equation above involves also the modulus of the torus $\tau$. Thus it can be used to convert the integrands for threshold corrections to differences of coupling constants into total derivatives on $\tau$-moduli space. We will focus on gauge couplings of $Z_{2} \times Z_{2}$ orbifold models. To derive such an equation we start from the integral expressions of such couplings (4.3) to obtain

$$
\Delta_{A B} \equiv \frac{16 \pi^{2}}{g_{A}^{2}}-\frac{16 \pi^{2}}{g_{B}^{2}}=-4 \mu^{3}\left(b_{A}-b_{B}\right) \int_{\mathcal{F}} \frac{d^{2} \tau}{\operatorname{Im} \tau^{2}} X^{\prime}(\mu) \operatorname{Im} \tau \Gamma_{2,2}(T, \bar{T}, U, \bar{U})
$$

\footnotetext{
*The special case for $d=2$ of this equation was noted and used in [9, 11].
} 
Eq. (5.4) does not apply to $U(1)$ 's that can get enhanced at special points of the moduli. Using (5.3) we obtain

$$
\left[\left(\mu \frac{\partial}{\partial \mu}\right)^{2}-2 \mu \frac{\partial}{\partial \mu}-16 \operatorname{Im} T^{2} \frac{\partial^{2}}{\partial T \partial \bar{T}}\right] \Delta_{A B}=0
$$

and we have also a similar one with $T \rightarrow U$. Note that for couplings that have a logarithmic behaviour, the double derivative of $\mu$ does not contribute.

We strongly believe that such equations also exist for single coupling constants using appropriate differential equations for $(d, d+n)$ lattices.

Notice that the IR scale $\mu$ plays the role of the RG scale in the effective field theory:

$$
\frac{16 \pi^{2}}{g_{A}^{2}(\mu)}=\frac{16 \pi^{2}}{g_{A}^{2}\left(M_{s t r}\right)}+b_{A} \log \frac{M_{s t r}^{2}}{\mu^{2}}+F_{A}\left(T_{i}\right)+\mathcal{O}\left(\mu^{2} / M_{s t r}^{2}\right)
$$

where the moduli $T_{i}$ have been rescaled by $M_{s t r}$ so they are dimensionless. Second, the IRFE gives a scaling relation for the moduli dependent corrections. Such relations are very useful for determining the moduli dependence of the threshold corrections. We will illustrate below such a determination, applicable to the $Z_{2} \times Z_{2}$ example described above.

Using the expansion (5.6) and applying the IRFE (5.5) we obtain

$$
\operatorname{Im} T^{2} \frac{\partial^{2}}{\partial T \partial \bar{T}}\left(F_{A}-F_{B}\right)=\frac{1}{4}\left(b_{A}-b_{B}\right)
$$

and a similar one for $U$. This non-homogeneous equation has been obtained in [9, 11].

Solving them we obtain

$$
F_{A}-F_{B}=\left(b_{B}-b_{A}\right) \log [\operatorname{Im} T \operatorname{Im} U]+f(T, U)+g(T, \bar{U})+\mathrm{cc}
$$

If at special points in moduli space, the extra massless states are uncharged with respect to the gauge groups appearing in (5.7) then the functions $f$ and $g$ are non-singular inside moduli space. In such a case duality invariance of the threshold corrections implies that

$$
F_{A}-F_{B}=\left(b_{B}-b_{A}\right) \log \left[\operatorname{Im} T \operatorname{Im} U|\eta(T) \eta(U)|^{4}\right]+\text { constant }
$$

This is the result obtained via direct calculation in [9].

It is thus obvious that the IRFE provides a powerful tool in evaluating general threshold corrections as manifestly duality invariant functions of the moduli.

\section{Further Directions}

Another set of important couplings that we have not explicitly addressed in this paper are the Yukawa couplings. Physical Yukawa couplings depend on the kähler potential and the

superpotential. The superpotential receives no perturbative contributions and thus can be calculated at tree level. The Khäler potential however does get renormalized so in order 
to compute the one-loop corrected Yukawa couplings we have to compute the one-loop renormalization of the Kähler metric. When the ground state has (spontaneously-broken) spacetime supersymmetry the wavefunction renormalization of the scalars $\phi_{i}$ are the same as those for their auxiliary fields $F_{i}$. Thus we need to turn on non-trivial $F_{i}$, calculate their effective action on the torus and pick the quadratic part proportional to $F_{i} \bar{F}_{\bar{j}}$. This can be easily done using the techniques we developed in this paper since it turns out that the vertex operators [23] for some relevant $F$ fields are bilinears of left and right U(1) chiral currents.

There are several open problems that need to be addressed in this context.

The structure of higher loop corrections should be investigated. A priori there is a potential problem, due to the dilaton, at higher loops. One would expect that since there is a region of spacetime where the string coupling become arbitrarily strong, higher order computations would be problematic. We think that this is not a problem in our models, because in Liouville models with $\mathrm{N}=4$ superconformal symmetry (which is the case we consider) there should be no divergence due to the dilaton at higher loops. However, this point need further study. One should eventually analyze the validity of non-renormalization theorems at higher loops [11] since they are of prime importance for phenomenology.

The consequences of string threshold corrections for low energy physics should be studied in order to be able to make quantitative predictions.

Finally, the response of string theory to the magnetic backgrounds studied in this paper should be analysed since it may provide with useful clues concerning the behavior of strings in strong background fields and/or singularities.

\section{Acknowledgements}

We would like to thank the organizers of the Spring School and Workshop on String Theory for giving us the opportunity to present our results. E. Kiritsis would like to thank ICTP for its hospitality during the workshop. C. Kounnas was supported in part by EEC contracts SC1*-0394C and SC1*-CT92-0789.

\section{References}

[1] P. Candelas, G. Horowitz, A. Strominger and E. Witten, Nucl. Phys. B258 (1985) 46.

[2] L.J. Dixon, J. Harvey, C. Vafa and E. Witten, Nucl. Phys. B261 (1985) 678; B274 (1986) 285;

K.S. Narain, M.H. Sarmadi and C. Vafa, Nucl. Phys. 288 (1987) 551.

[3] K.S. Narain, Nucl. Phys. B169 (1986) 41;

K.S. Narain, M.H. Sarmadi and E. Witten, Nucl. Phys. B279 (1987) 369;

[4] W. Lerche, D. Lüst and A.N. Schellekens, Phys. Lett. B181 (1986) 71;

Nucl. Phys. B287 (1987) 477. 
[5] I. Antoniadis, C. Bachas and C. Kounnas, Nucl. Phys. B289 (1987) 87;

H. Kawai, D.C. Lewellen and S.H.-H. Tye, Nucl. Phys. B288 (1987) 1.

[6] W. Lerche, D. Lüst and A.N. Schellekens, Phys. Lett. B181 (1986) 71; Nucl. Phys. B287 (1987) 477.

[7] D. Gepner, Phys. Lett. B199 (1987) 370; Nucl. Phys. B296 (1988) 757.

[8] E. Witten, Phys. Lett. B155 (1985) 151;

S. Ferrara, C. Kounnas and M. Porrati, Phys. Lett. B181 (1986) 263;

S. Ferrara, L. Girardello, C. Kounnas and M. Porrati, Phys. Lett. B193 (1987) 368;

I. Antoniadis, J. Ellis, E. Floratos, D.V. Nanopoulos and T. Tomaras, Phys. Lett. B191 (1987) 96;

S. Ferrara, L. Girardello, C. Kounnas and M. Porrati, Phys. Lett. B194 (1987) 358;

M. Cvetic, J. Louis and B. Ovrut, Phys. Lett. B206 (1988) 227;

M. Cvetic, J. Molera and B. Ovrut, Phys. Rev. D40 (1989) 1140;

L. Dixon, V. Kaplunovsky and J. Louis, Nucl. Phys. B329 (1990) 27;

M. Cvetic, B. Ovrut and W. Sabra, hep-th/9502144.

[9] V.S. Kaplunovsky, Nucl. Phys. B307 (1988) 145;

L.J. Dixon, V.S. Kaplunovsky and J. Louis, Nucl. Phys. B355 (1991) 649.

[10] J.-P. Derendinger, S. Ferrara, C. Kounnas and F. Zwirner, Nucl. Phys. B372 (1992) 145 and Phys. Lett. B271 (1991) 307;

G. Lopez Cardoso and B.A. Ovrut, Nucl. Phys. B369 (1992)351;

S. Ferrara, C. Kounnas, D. Lüst and F. Zwirner, Nucl. Phys. B365 (1991) 431.

[11] I. Antoniadis, K. Narain and T. Taylor, Phys. Lett. B276 (1991) 37;

I. Antoniadis, E. Gava and K. Narain Phys. Lett. B283 (1992) 209; Nucl. Phys. B383 (1992) 93;

I. Antoniadis, E. Gava, K.S. Narain and T. Taylor, Nucl. Phys. B407 (1993) 706 hepth/9212045); ibid. B413 (1994) 162 (hep-th/9307158); ibid. Nucl. Phys. B432 (1994) 187 (hep-th/9405024).

[12] C. Kounnas, Phys. Lett. B321 (1994) 26; Proceedings of the International "LeptonPhoton Symposium and Europhysics Conference on High Energy Physics", Geneva, 1991, Vol. 1, pp. 302-306; Proceedings of the International Workshop on "String Theory, Quantum Gravity and Unification of Fundamental Interactions", Rome, 21-26 September 1992.

[13] E. Kiritsis, in the Proceeding of the International Europhysics Conf. on HEP, Marseille, 1993, Eds. J. Carr and M. Perrotet (hep-th/9309064).

[14] I. Antoniadis, S. Ferrara and C. Kounnas Nucl. Phys. B421 (1994) 343.

[15] E. Kiritsis and C. Kounnas, Phys. Lett. B331 (1994) 51 (hep-th/9404092). 
[16] P. Mayr and S. Stieberger, hep-th/9303017, hep-th/9304055, hep-th/9504129;

P. Mayr, H. Nilles and S. Stieberger, hep-th/9307171;

G. Lopes Cardoso, D. Lüst and B. Ovrut, hep-th/9410056;

V. Kaplunovsky and J. Louis, hep-th/9402005, hep-th/9502077;

G. Lopes Cardoso, D. Lüst and T. Mohaupt, hep-th/9412071;

B. de Wit, V. Kaplunovsky, J. Louis and D. Lüst, hep-th/9504006;

I. Antoniadis, S. Ferrara, E. Gava, K. Narain and T. Taylor, hep-th/9504034;

K. Dienes and A. Faraggi, hep-th/9505018, hep-th/9505046.

[17] J. Minahan, Nucl. Phys. B298 (1988) 36.

[18] I. Antoniadis, C. Bachas, J. Ellis and D. Nanopoulos, Nucl. Phys. B328 (1989) 117.

[19] E. Kiritsis and C. Kounnas, Nucl. Phys.

B442 (1995) 472, (hep-th/9501020);Nucl. Phys. B41 [Proc. Sup.] (1995) 331, hepth/9410212).

[20] E. Kiritsis and C. Kounnas, hep-th/9508078.

[21] I. Antoniadis C. Bachas and A. Sagnotti, Phys. Lett. B235 (1990) 255;

C. Bachas and E. Kiritsis, Phys. Lett. B325 (1994) 103 (hep-th/9311185).

[22] J. Russo and A. Tseytlin, hep-th/9411099, hep-th/9502038;

A. Tseytlin, Phys. Lett. B346 (1995) 55 (hep-th/9411198).

[23] J. Attick, L. Dixon and A. Sen, Nucl. Phys. B292 (1987) 109. 